\title{
Analysis of microwave polarization difference index characteristics about different vegetation types in northeast of China
}

\author{
Yang-yang $\mathrm{Li}^{1,2}$, Kai Zhao ${ }^{1 *}$, Xing-ming Zheng ${ }^{1}$, Jian-hua Ren ${ }^{1,2}$ \\ 1. Northeast Institute of Geography and Agroecology, Chinese Academy of Sciences, Changchun, China \\ 2. University of Chinese Academy of Sciences, Beijing, China
}

\begin{abstract}
Microwave polarization difference index (MPDI) has great potential in soil moisture content and biomass inversion. Passive microwave remote sensing can be used for inversing surface parameters such as surface temperature, vegetation water content. Different terrain types have different polarization effects and radiation characteristics, and also present the differences by seasons due to its structure, moisture content, and roughness. Use monthly AMSR-E microwave brightness temperature data $(25 \mathrm{Km})$ under clear sky in 2010 and IGBP classification standard data $(1 \mathrm{KM})$, then analyses the MPDI characteristics and the MPDI season change rule of different surface types by choosing relatively pure pixels. Result shows that, MPDI has relationship with frequency, almost all MPDI of vegetations, water, bare soils decreased along with the increase frequency. In addition, MPDI have difference by seasons of different vegetation types, which has relationship with vegetation density and canopy characteristic; Snow cover also is a important factor in Northeast of China.
\end{abstract}

Keywords-Microwave polarization difference index(MPDI); vegetation types; AMSR-E;soil moisture content

\section{INTRODUCTION}

Vegetation index is widely used in optical remote sensing, according to the statistics, there are more than 40 indexes are proposed in visible and infrared remote sensing [1]. With the development of microwave technology, the appearance and the thorough application promoted the further development of microwave remote sensing in surface parameter retrieval [2]. Microwave index is proposed first by Paloscia [3], after that Choudhury, Calvet et al also did many researches about microwave index.[4,5,6] Research results show that different vegetation types have a deep influences on microwave index and also the vegetation water content. That conclusion is wide used in soil moisture retrieval, especially in vegetation covered area. So it is very important to analyze the MPDI characteristics about different vegetation types. This paper based on the analysis expression of MPDI,

\section{CONCEPT AND ANALYSIS ABOUT MPDI}

Layers of vegetation are an important factor which affects the surface emission, and can be described as a scatter layers above a rough soil. Vegetation has both an attenuation effect and increase effect on surface radiation. MPDI is based on microwave radiation transfer equation. In the perspective of electromagnetic wave propagation, vegetation is composed of discrete vegetation dielectric materials (leaves, trunk, and fruit, etc) and air [7]. In most of the canopy, the vegetation dielectric material has a nearly size or greater than the wavelength in microwave band which means the canopy is heterogeneous and anisotropic medium. Electromagnetic wave will have absorption and scattering when penetrates this kind of medium [8].Usually use $\omega-\tau$ radiative transfer model to represent the vegetation coverage soil microwave radiation in equation (1):

$$
T_{b p}(\tau, \mu)=(1-\omega)\left(1-e^{-\tau / \mu}\right)\left(1+e^{-\tau / \mu}\right) T_{v e g}+T_{\text {soil }} e^{-\tau / \mu}
$$

Where, $T_{b p}(\tau, \mu)$ is the brightness temperature in the angle of $\theta, \mathrm{p}$ is polarization mode, $\mu=\cos (\theta), \tau$ is the optical thickness, $\omega$ is the single scattering albedo.

If we set the vegetation and soil as an integral, then the emission is polarized. Due to the depolarization effects of vegetation on a single signal launch, MPDI can be used to describe the characterization of vegetation. Usually the MPDI is defined in equation (2) [3]:

$$
\operatorname{MPI}(\tau, \mu)=\frac{T_{b V}-T_{b H}}{T_{b V}+T_{b H}}
$$

The equation (1) generation into the equation (2):

$$
\operatorname{MPI}(\tau, \mu)=\frac{\left(\varepsilon_{V}-\varepsilon_{H}\right) e^{-\tau / \mu} T_{\text {soil }}+(1-\omega)\left(1-e^{-\tau / \mu}\right) e^{-\tau / \mu} T_{\text {veg }}\left(R_{V}-R_{H}\right)}{(1-\omega)\left(1-e^{-\tau / \mu}\right) T_{\text {veg }}\left(2+e^{-\tau / \mu}\left(R_{V}-R_{H}\right)\right)+\left(\varepsilon_{V}+\varepsilon_{H}\right) e^{-\tau / \mu} T_{\text {soil }}}
$$

For bare soil, $\tau=0$, while the MPDI can be described in equation (4):

$$
\operatorname{MPI}(0, \mu)=\frac{\left(\varepsilon_{V}-\varepsilon_{H}\right)}{\left(\varepsilon_{V}+\varepsilon_{H}\right)}
$$

Assume that the temperature of vegetation is equal to the soil, the relationship of MPDI between bare soil and vegetation covered surface shows in equation (5):

$$
\operatorname{MPI}(\tau, \mu)=\operatorname{MPI}(0, \mu) \frac{\left(\varepsilon_{H}+\varepsilon_{V}\right) e^{-\tau / \mu}\left(1-(1-\omega)\left(1-e^{-\tau / \mu}\right)\right)}{2(1-\omega)\left(1-e^{-\tau / \mu}\right)\left(1+e^{-\tau / \mu}\right)+\left(\varepsilon_{H}+\varepsilon_{V}\right) e^{-\tau / \mu}\left(1-(1-\omega)\left(1-e^{-\tau / \mu}\right)\right)}
$$

the MPDI between vegetation covered and bare soil has a complicated relationship shows the above expression. If the part energy of downward radiation scattering reflected and then attenuated by vegetation is not considered, that means $e^{-\tau / \mu} R_{p}$ can be omitted, then:

$$
\operatorname{MPI}(\tau, \mu) \approx \operatorname{MPI}(0, \mu) e^{-\tau / \mu}
$$


we can know that, MPDI is mainly determined by $\tau$ and $\mu$, which $\tau$ depends mainly on vegetation water content. In the equation (5), the emissivity is determined by the dielectric constant, generally, vegetation materials can be the mixture of plant corpus and water. The dielectric constant of plant corpus, can be gained from measurement, $1.5 \leq \varepsilon_{\mathrm{r}}{ }^{\prime} \leq 2.0$ and $\varepsilon_{\mathrm{r}}{ }^{\prime \prime} \leq 0.1[8]$, however the water is about 81 , thus vegetation dielectric constant is mainly affected by water content. Different vegetations, for the differences of canopy shape, leaf structure, and plant growth result to a large difference about the vegetation water content, meanwhile, the soil moisture content affects the vegetation water content and the optical thickness of vegetation is influenced by vegetation water content, to some extent, MPDI can reflect the vegetation water content and then indirectly reflect the soil moisture content especially in vegetation covered area.

\section{AVAILABLE DATA}

The earth observation satellite Aqua with passive microwave sensor AMSR-E and optical sensor MODIS, which provides a convenient for using multi-source remote sensing data comprehensively. We have synthesis vegetation index products with 16 days. AMSR-E, have 6 bands during $6.9 \mathrm{GHz}$ to $89 \mathrm{GHz}$ with a resolution of $25 \mathrm{Km}$ and two polarization mode. Take northeast of China as an example, in order to carry out the analysis of MPDI with different vegetation types and also reduce the influence of mixed pixels, a combination of the LUCC with a $1 \mathrm{Km}$ resolution according to IGBP is used, shows in Fig.1. The percentage on surface type of LUCC is higher than $85 \%$ within a AMSR-E pixel will be chose as an pure pixel and then extracted to further analysis. The brightness temperatures of all bands and each month are extracted, and then MPDI is calculated using equation (2):

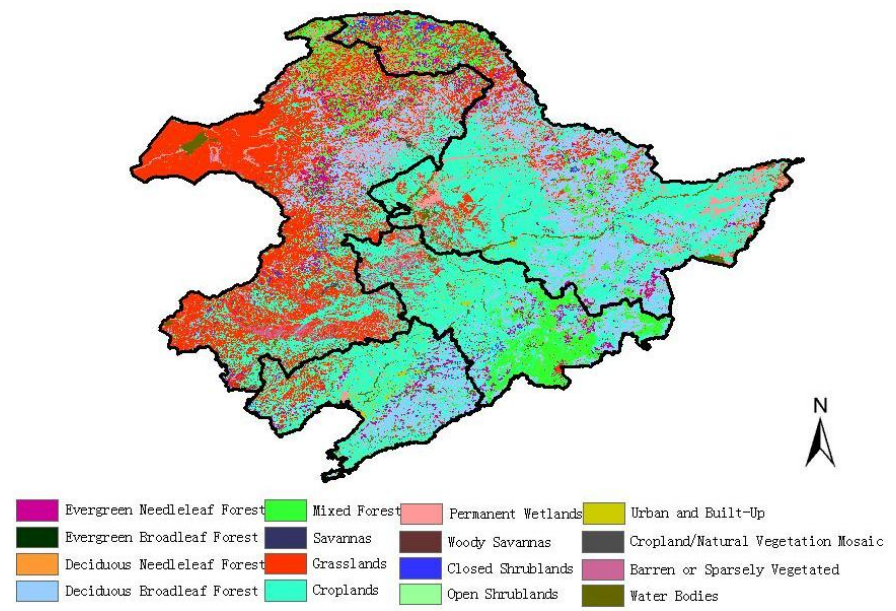

Figure 1. Classification map of northeast China(2006 IGBP).

\section{RESUlTS ANALYSIS}

\section{A. Relationship between MPDI and Frequency}

Fig. 2 shows the MPDI variation trend of 9 types of different features along with the change of frequency in July $8^{\text {th }} 2010$. Vegetation grows the most flourishing in July, and it is benefits to analyze the vegetation types through MPDI. The horizontal axis is frequency $(\mathrm{GHz})$ while the vertical is MPDI. Different surface features have different polarization effect. Generally, the MPDI is positive, that means V-polarization is higher than $\mathrm{H}$-polarization, which conforms to the theory analysis. For each feature show in Fig.2, MPDI decreases with the frequency increase. The reason is that for vegetation the volume scattering enhances especially in dense forests while the frequency increases, and the vegetation scattering is directional, both of which is depolarization effect, and then the MPDI decreases. That is why MPDI is lower for needle leaved forest and broad leaved forest than grass land and savanna, shows in Fig.1 (f) and (g) with (b) and (c). The surface roughness is also the important factor which affects microwave radiation, smooth surface generally has higher MPDI than roughness surface.
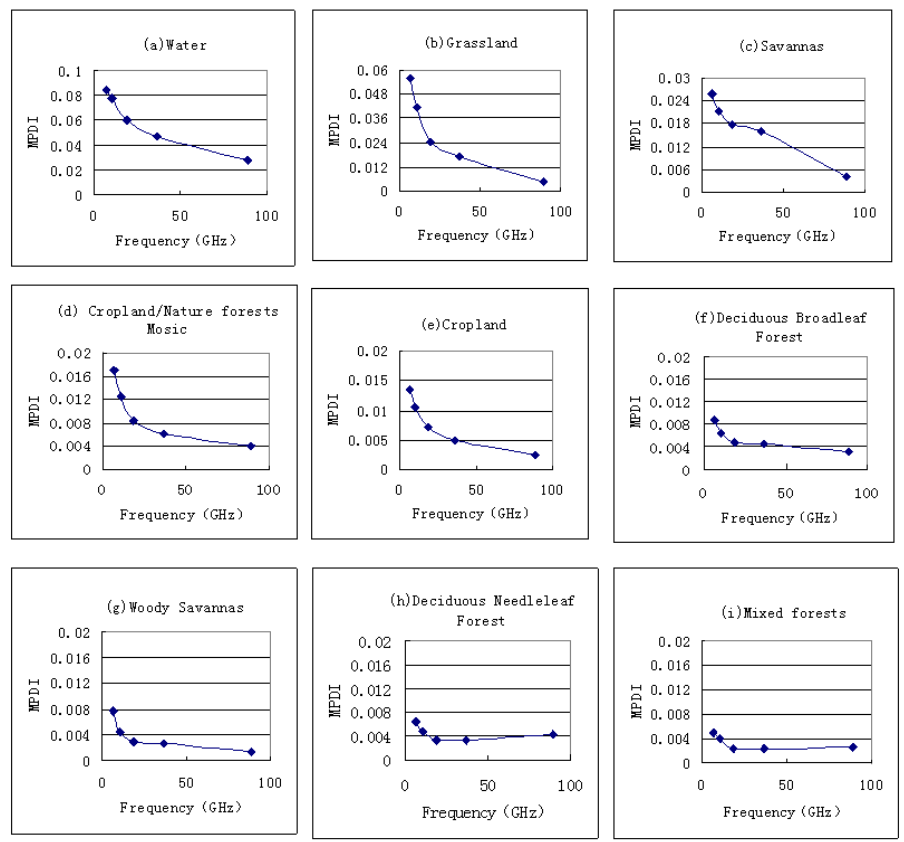

Figure 2. MPDI on frequency change of different surface types

\section{B. MPDI Analysis with Time Series}

Choose several features with high pixel purity, and then analyze the change rule of MPDI with time series, results show in Fig.3. The horizontal axis is time by month while the vertical is MPDI. The MPDI of each month is extract by a random average data of 7 days in this month at $6.9 \mathrm{GHz}$. In winter (middle October to March of the following year) the 


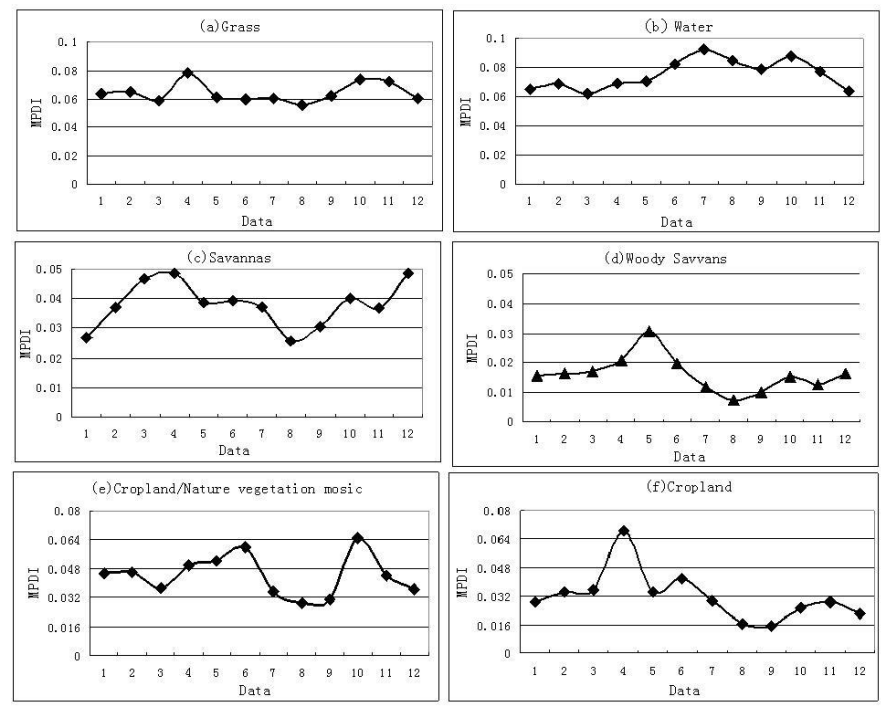

Figure 3. MPDI on frequency change of different surface types

MPDI is relatively a little low to other months. This is due to that the surface is covered by snow in most of the time in northeast of China, and the coverage with snow result in a large amount of scattering which increases the contribution of $\mathrm{H}$ polarization and then reduce the MPDI. As the snow began to melt, MPDI has increasing trend and until April or May is the largest of the whole year. After that, due to growth of vegetation, the depolarization effect became obvious.

\section{CONCLUSION AND DISCUSSION}

Research of MPDI is paid more and more attention in recent years. MPDI has a great relevance with vegetation types and soil moisture. It can be used to describe the change of surface, especially for vegetation parameters, such as vegetation optical thickness. With its all-weather, all-time capabilities and the penetrability, microwave remote sensing both passive and active has great potential for forestry management. According to the previous analysis, the following conclusions can be proposed:

(1) MPDI decreases with the increment of frequency. The more complex vegetation canopy, such as cropland or forest, the difference of MPDI on frequency is smaller. This shows that the impact of vegetation is more sensitive in low frequency and it is available to take the advantage of low frequency data on the inversion of vegetation information.

(2) The MPDI varies with seasonal change for different surface type. Generally speaking, the difference of MPDI is small in the summer and fall seasons which vegetation grow well at this time. The dense canopy and high plant result to an obvious depolarization. That means different vegetation types have different canopy structure and plant height which may available for MPDI to reflect the vegetation growth and other related information.

(3) In winter, snow coverage is an important factor. The scattering of snow and the trunk of plant both have effects on the microwave radiation and reduce the MPDI. Most of the vegetation in winter in northeast of China are deciduous forest and only have trunk in this season, except for needle leaved forest. It is easy for microwave band to penetrate the trunk and reach to the snow layer, and all of which decrease the MPDI.

This paper analyzed the MPDI in northeast of China with different surface coverage types. Study shows that vegetation types are very important for microwave radiation and then reflect in MPDI. It is need to be considered for soil moisture or snow parameters inversion in vegetation coverage areas by microwave remote sensing. For the low resolution of passive microwave sensors, there is a combination contribution for one pixel by a variety of features. Research of MPDI with different surface types provided the possibility for decomposition of mixed pixels of microwave remote sensing.

\section{ACKNOWLEDGMENT}

Thanks to the Laboratory of Remote Sensing and Geographic Information Science of Institute of Cold and Arid Regions Environmental and Engineering Research for providing the $1 \mathrm{~km}$ land cover classification data.

\section{REFERENCES}

[1] Q.Tian, X.Min, "Advances in study on vegetation indices," Advance in Earth Sciences., vol. 13, no.4, pp.327-333, Aug.,1998.

[2] F.Gao, T.Che,J.Wang, and J.Wen, "Passive microwave remote sensing indexes and their application," Remote Sensing Technology and Application., vol.20, no.6,pp.551-557,Dec., 2005.

[3] S. Paloscia, P.Pampaloni, "Experiment relationships between microwave emission and vegetation features," Int. J. Remote sensing., vol.6, pp.315-323., 1985.

[4] B.J.Choudhury,C.J.Tucker, "Monitoring vegetation using Nimbus7 scanning multichannel microwave radiometer's Data," Int.J.Remote Sensing., vol.8,no.7,pp.533-588,1987.

[5] B.J.Choudhury,C.J.Tucker,"Monitoring vegetation using Nimbus-7 $37 \mathrm{GHz}$ data :some empirical relations," Int.J.Remote Sensing., vol.8,no.7,pp.1085-1090,1987

[6] J.C.Calvet, J.P.Wigneron,E.Mougin, "Plant water content and temperature of the Amazon forest from satellite microwave radiometry,' IEEE Transations on Geoscience and Remote Sensing., vol.32,pp.397-408,1994.

[7] J.C.Shi,T.Jackson,J.Tao, "Microwave vegetation indices for shor vegetation covers from satellite passive microwave sensor AMSR-E," Remote Sensing of Environment.,vol.112,pp.4285-4300,2008.

F.T.Ulaby,R.K.Moore, "Microwave remote sensing:active and passive,vol.III-volume scattering and emission theory, advanced systems and applications, Dedham,1987,ch.19. 